\title{
METODOLOGIA E SUFICIÊNCIA AMOSTRAL EM ESTUDOS ETNOBOTÂNICOS E ETNOFARMACOLÓGICOS: RELATO DE EXPERIÊNCIA
}

\author{
METHODOLOGY AND SAMPLE SUFFICIENCY IN ETHNOBOTANICAL AND \\ ETHNOPHARMACOLOGICAL STUDIES: EXPERIENCY REPORT
}

\author{
GONÇALVES'1, Rodrigo Noll; GONÇALVES², Jéssica Rodrigues da Silva Noll; \\ BUFFON $^{3}$, Marilene da Cruz Magalhães
}

\begin{abstract}
1 - Mestre em Saúde Coletiva pelo Programa de Pós-Graduação em Saúde Coletiva UFPR
2 - Mestranda em Saúde Coletiva pelo Programa de Pós-Graduação em Saúde Coletiva UFPR

3 - Professora Doutora do Programa de Pós-Graduação em Saúde Coletiva UFPR

Autor para correspondência: rodrigo.noll@hotmail.com
\end{abstract}

\section{RESUMO:}

Os estudos etnobotânicos e etnofarmacológicos constituem importantes estratégias que podem contribuir para a descoberta de novos fármacos. A curva de acumulação de espécies (ou curva do coletor) vem sendo amplamente utilizada no Brasil, constituindo um método quantitativo para determinar se a amostra é representativa da comunidade em estudo. O presente artigo aborda a curva de acumulação de espécies como uma estratégia metodológica em estudos etnobotânicos e etnofarmacológicos. Para tal, apresenta-se um relato de experiência, demonstrando o procedimento metodológico aplicado em levantamento etnobotânico e etnofarmacológico realizado na área rural do município de Campo Largo (Paraná, Brasil), onde foi empregada a curva de acumulação de espécies na determinação da suficiência amostral, a fim de contribuir com estudos posteriores.

Palavras-chave: Etnobotânica; Etnofarmacologia; Plantas Medicinais.

\section{ABSTRACT:}

Ethnobotanical and ethnopharmacological studies are important strategies that can contribute to the discovery of new drugs. The species accumulation curve (or curve of the collector) has been widely used in Brazil, constituting a quantitative method to determine if the sample is representative of the study community. This article discusses the species accumulation curve as a methodological strategy in ethnobotanical and ethnopharmacological studies. For this, an experience report is presented, demonstrating the methodological procedure applied an ethnobotanical and ethnopharmacological survey carried out in the rural area of the municipality of Campo Largo (Paraná, Brazil), where the species accumulation curve was used to determine the sample sufficiency, in order to contribute to further studies.

Key-words: Ethnobotany; Ethnopharmacology; Medicinal Plants.

\section{INTRODUÇÃO}

O Brasil é o país que possui a maior biodiversidade do planeta que, associada a uma rica diversidade étnica e cultural, detém um valioso conhecimento tradicional associado ao uso de plantas medicinais, e possui o potencial necessário para desenvolvimento de 
pesquisas com resultados em tecnologias e terapêuticas apropriadas (BRASIL, 2006a).

Dentre as inúmeras contribuições do conhecimento popular, o uso de plantas medicinais constitui uma forma de tratamento com origens muito antigas, relacionada aos primórdios da medicina e fundamentada no acúmulo de informações por sucessivas gerações. Ao longo dos séculos, produtos de origem vegetal constituíram as bases para o tratamento de diferentes doenças (BRASIL, 2015).

A biodiversidade brasileira inspira o uso de plantas medicinais e seus derivados, fortalecendo a implantação dos programas desenvolvidos na área da fitoterapia, que surgiram com o intuito de parametrizar diretrizes e incentivar a pesquisa no setor de plantas medicinais e fitoterápicos (LIMA; GOMES, 2014).

A cadeia produtiva de plantas medicinais e fitoterápicos apresenta interface com diversas áreas do conhecimento, e demandam, portanto, ações multidisciplinares (BRASIL, 2006b). Estudos multidisciplinares envolvendo "a etnobotânica, a química e a farmacologia" apresentam grande importância para o enriquecimento dos conhecimentos sobre produtos de origem vegetal (MACIEL et al., 2002, p. 430).

Dentre as possibilidades metodológicas, a curva de acumulação de espécies (ou curva do coletor) constitui importante estratégia a ser aplicada na determinação da suficiência amostral. Neste contexto, o presente estudo baseia-se um relato de experiência sobre o procedimento metodológico aplicado em levantamento etnobotânico e etnobotânico realizado na área de abrangência de uma unidade de saúde da família (USF) rural do município de Campo Largo (Paraná, Brasil), onde foi empregada a curva de acumulação de espécies na determinação da suficiência amostral. Adicionalmente, apresenta-se as principais características e descrição do procedimento metodológico, visando contribuir para a realização de novos estudos etnobotânicos e etnofarmacológicos.

\section{METODOLOGIA}

Este artigo baseia-se em um relato de experiência sobre o procedimento metodológico aplicado em levantamento etnobotânico e etnofarmacológico sobre o uso de plantas medicinais realizado junto à comunidade da área de abrangência da USF Itambezinho, localizada na área rural do município de Campo Largo (Paraná, Brasil) (GONÇALVES, 2017).

O estudo seguiu as normas da Resolução 466/12 do Conselho Nacional de Saúde e teve início após a aprovação do projeto pelo Comitê de Ética em Pesquisas (CEP) do 
Setor de Ciências da Saúde da Universidade Federal do Paraná (UFPR), parecer CEP/SDPB nำ1.343.161, de 30 de novembro de 2015.

O universo amostral correspondeu a 31 famílias residentes na área de abrangência da USF, aleatoriamente selecionadas entre aquelas previamente identificadas como usuárias de plantas medicinais, por meio das fichas do sistema e-SUS Atenção Básica, utilizadas em visitas domiciliares pelos Agentes Comunitários de Saúde. A suficiência amostral foi determinada pela curva de acumulação de espécies.

\section{CARACTERIZAÇÃO DO ESTUDO}

A coleta de dados deu-se por entrevistas semiestruturadas realizadas de 15 de dezembro de 2015 a 29 de julho de 2016, com o membro da família maior de idade que estava presente na residência e se disponibilizava para tal atividade.

Após apresentação e explanação inicial acerca do objetivo da pesquisa, e concordância do entrevistado com assinatura do Termo de Consentimento Livre e Esclarecido (TCLE), a entrevista era iniciada. Utilizou-se roteiro básico previamente testado quanto à validade e confiabilidade (GIL, 2002).

Além da informação sobre a identificação pessoal (nome completo, endereço, sexo, idade, escolaridade, ocupação, renda familiar, dados sobre os membros da família, dados sobre o tipo de residência), durante a entrevista foram anotados dados sobre plantas medicinais utilizadas com finalidade terapêutica, modo de uso, finalidade e fonte de obtenção deste conhecimento, além dos nomes dos medicamentos de uso contínuo utilizados pelas famílias entrevistadas.

$\mathrm{Na}$ análise de dados, as indicações de uso popular foram substituídas pelas propriedades terapêuticas correspondentes, por exemplo: para "dor", utilizou-se "analgésico"; para "inflamação", utilizou-se "anti-inflamatório"; para "infecção", utilizou-se "antimicrobiano" (INFOPÉDIA, 2017).

Sempre que possível, realizou-se a coleta e herborização (atividade que antecede o processo de incorporação do material no herbário) das plantas citadas, segundo Fidalgo e Bononi (1989), para preparo da exsicata:

Para tal finalidade, foi realizada a coleta de exemplares de no máximo 30 a $40 \mathrm{~cm}$., e quando possível, da planta inteira. Os ramos foram cortados, sempre que possível, juntamente com folhas, flores e frutos. Coletados os exemplares, estes receberam etiqueta de identificação numérica, correspondente ao número do registro, e foram arrumados de 
forma a reproduzir a posição vista no campo, distendidos em folhas inteiras e dobradas de jornal colocadas entre papelão canelado com os canais orientados sempre no mesmo sentido, de modo a constituir um empilhado entre dois cartões resistentes. O conjunto foi prensado firmemente por duas cordas, formando as prensas para transporte.

No campo, o processo de secagem iniciou-se nas próprias prensas. Em seguida, realizou-se troca diária das folhas de jornal umedecidas por outras secas, e as prensas foram expostas ao sol, por 5 a 6 dias no máximo, em posição tal que favorecia a penetração dos raios solares. Para acelerar a secagem, o material foi transferido assim que possível para estufa com circulação de ar quente, por 2 a 3 dias. Antes de levar as prensas à estufa, foram colocadas chapas de alumínio corrugado entre as folhas de papelão canelado com o sentido do corrugado acompanhando o dos canais do papelão e as prensas foram colocadas na estufa em posição que favorecia a circulação de ar. A Figura 1 apresenta um exemplo de exsicata obtida após os procedimentos de secagem.

Figura 1. Exsicata dobrada e arrumada de uma planta inteira

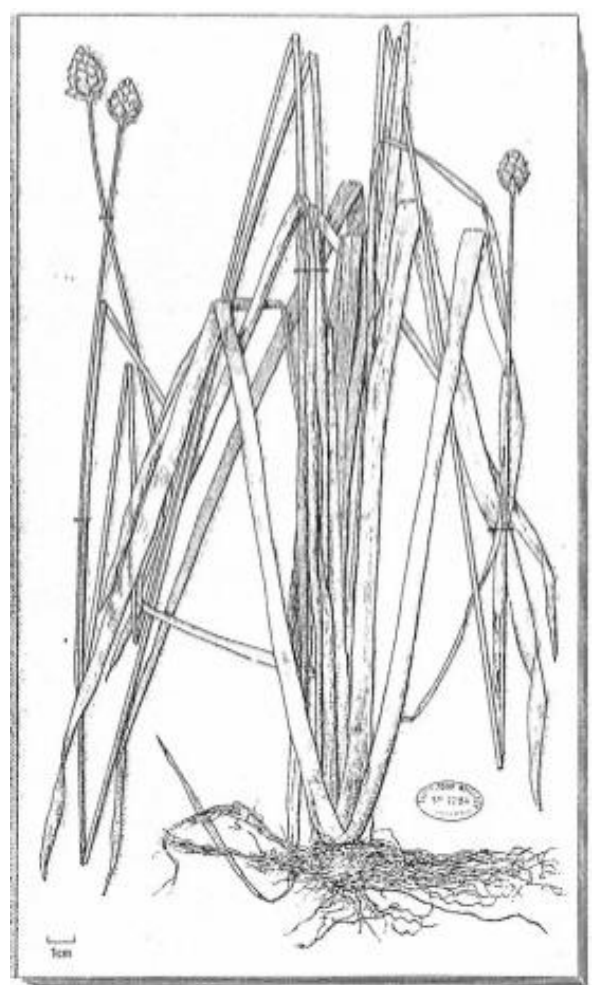

FONTE: FIDALGO; BONONI, 1989.

A identificação do material coletado seguiu os padrões da taxonomia clássica, feita com base em caracteres morfológicos vegetativos e florais e utilizando-se, quando possível, vários exemplares. As determinações foram efetuadas por meio de chaves analíticas e 
comparações com materiais depositados no Herbário do Departamento de Botânica da UFPR (UPCB), e no Herbário do Museu Botânico Municipal (MBM) de Curitiba (PR). A confirmação das determinações foi efetuada por equipe técnica do MBM.

As exsicatas correspondentes ao mesmo nome científico foram agrupadas e o conjunto recebeu etiqueta contendo a família da planta, nome científico e data da identificação.

As informações etnobotânicas coletadas foram analisadas comparativamente ao exposto em distintas fontes impressas e eletrônicas, no sentido de identificar incongruências quanto a indicações de usos e também riscos de utilização inapropriada.

\section{ESTUDOS ETNOBOTÂNICOS E ETNOFARMACOLÓGICOS}

O estudo de práticas terapêuticas provenientes de diferentes povos e culturas pode fornecer informações para descoberta de novos fármacos de interesse médico e farmacêutico (ALBUQUERQUE; HANAZAKI, 2006).

Segundo Albuquerque e Hanazaki (2006, p. 678), "as investigações etnofarmacológicas e etnobotânicas têm sido a principal abordagem reconhecida por cientistas em todo o mundo, como uma estratégia de seleção de plantas medicinais".

A etnobotânica e a etnofarmacologia são áreas onde se buscam informações a partir do conhecimento de diferentes povos e etnias. Neste caso, a etnobotânica inclui as formas de percepção e apropriação dos recursos vegetais pelos povos (ALBUQUERQUE; HANAZAKI, 2006). Já a etnofarmacologia trata do conhecimento popular relacionado aos sistemas tradicionais de medicina (ELISABETSKY, 2003), objetivando a identificação e o registro dos diferentes usos medicinais de espécies de plantas por diferentes grupos humanos (DI-STASI, 2005).

\section{DETERMINAÇÃO DA SUFICIÊNCIA AMOSTRAL}

A mensuração da riqueza de espécies é um objetivo essencial para muitas comunidades ecológicas e para a conservação biológica. O número montante de espécies em um local é um índice intuitivo e natural de estrutura da comunidade. Dessa forma, os padrões de riqueza de espécies têm sido medidos em pequenas e largas escalas espaciais (GOTELLI; COLWELL, 2010).

A determinação da variação das espécies em comunidades apenas será alcançada 
quando a amostragem for representativa de toda a área. No entanto, devido ao cronograma temporal, ou ao "orçamento elevado, a alternativa é fazer uso de procedimentos de amostragem". Em estudos florísticos, a suficiência amostral pode ser obtida por meio de distintos métodos, entre eles, a curva espécie-área (GOMIDE et al., 2005).

Segundo Kersten \& Galvão (2011, p. 156), a amostra pode ser definida como

"[...] o subconjunto de uma população por meio do qual se estabelecem ou estimam as propriedades ou características dela. Assim, uma amostra não é a população, mas espera-se que a represente o mais fielmente possível $[\ldots]^{\prime \prime}$.

Segundo Schilling et al. (2012, p. 101-102),

"A principal dificuldade nos estudos que visam estimar a riqueza de espécies de um local é determinar o quanto é necessário amostrar para se obter uma previsão acurada dessa variável, pois é importante saber se a amostra é grande o suficiente para permitir estimar com precisão a riqueza de espécies de uma comunidade que, muitas vezes, não tem tamanho conhecido".

Por não contemplar adequadamente o ambiente em estudo, a deficiência amostral pode proporcionar interpretações e conclusões equivocadas da fisionomia estudada. "Isso impede a realização de comparações estruturais, florísticas e de diversidade influenciando decisivamente na análise da vegetação". Ou seja, "somente após a determinação da suficiência amostral, pode-se [...] proceder à quantificação de vários índices de diversidade e similaridade", para obter conclusões sobre as peculiaridades da vegetação amostrada (GOMIDE et al., 2005, p. 377).

\section{A CURVA DE ACUMULAÇÃO DE ESPÉCIES}

Segundo Schilling e Batista (2008), a curva de acumulação de espécies, ou curva do coletor, consiste na representação gráfica utilizada na determinação da suficiência amostral. Ainda, segundo os autores,

"A definição de um tamanho ótimo de amostra está baseado na ideia de que quanto maior o tamanho da amostra, maior o número de espécies que será 
encontrado, mas a uma taxa decrescente, até o ponto em que a curva estabiliza e torna-se horizontal. Esse ponto seria a área mínima necessária para representar a comunidade" (SCHILLING; BATISTA, 2008, p. 179).

De acordo com Wandscheer et al. (2016), este método amostral vem sendo amplamente utilizado no Brasil, sendo uma forma quantitativa para determinar se a amostra utilizada é representativa da comunidade em estudo. Silveira et al. (2010) considera que a melhor forma de se avaliar o esforço de amostragem ocorre por meio da curva de acumulação de espécies.

A curva de acumulação de espécies "é uma técnica que surgiu da relação espécieárea, considerada de grande importância na caracterização de comunidades vegetais, e que vem sendo extensivamente utilizada em estudos de fitossociologia", especialmente no Brasil, na determinação da suficiência amostral (SCHILLING; BATISTA, 2008, p. 179).

O "gráfico consiste no número de espécies (grafado no eixo das ordenadas ou eixo y)", e "o esforço amostral (grafado no eixo das abscissas ou eixo x)". O esforço amostral pode ser medido de várias formas, tais como o "número de unidades amostrais, número de indivíduos amostrados, horas de observação", entre outras (BATISTA; SCHILLING, 2006, p. 2).

Silva (2012) constatou, em estudo, que a curva de acumulação de espécies é uma importante ferramenta utilizada na adequação do esforço amostral, pois fornece subsídios que auxiliam nas tomadas de decisões, considerando a riqueza taxonômica e o estabelecimento de estratégias para conservação da biodiversidade.

\subsection{Aplicação prática da curva de acumulação de espécies}

No estudo realizado junto à comunidade da área de abrangência da USF Itambezinho (GONÇALVES, 2017), foram entrevistadas 31 famílias, aleatoriamente selecionadas entre aquelas previamente identificadas como usuárias de plantas medicinais, por meio das fichas do sistema e-SUS Atenção Básica, utilizadas em visitas domiciliares pelos Agentes Comunitários de Saúde.

No período do estudo, a USF prestava atendimento a uma população de 346 famílias, distribuídas em quatro microáreas, totalizando 25 comunidades rurais. A densidade populacional entre as comunidades rurais era bastante diversificada, sendo "Itambezinho" o maior núcleo populacional com 80 famílias, e "Passa Três" o menor, com 2 famílias. 
Foram registradas 426 citações de plantas medicinais, totalizando um escopo de 120 espécies identificadas. A curva foi construída com base em quantas plantas diferentes foram identificadas na família entrevistada, em relação a quantidade de plantas identificadas até a entrevista anterior, da seguinte forma: na Família 1, foram identificadas 25 plantas distintas, totalizando 25 plantas acumuladas; na Família 2, foram identificadas 13 plantas distintas, totalizando 38 plantas acumuladas; na Família 3, foram identificadas 18 plantas distintas, totalizando 56 plantas acumuladas; e assim sucessivamente, até que na Família 26, o número de plantas acumuladas atingiu 120. Este valor manteve-se estável por mais 5 famílias entrevistadas, caracterizando a estabilização da curva, que se tornou horizontal, determinando a suficiência amostral, conforme demonstrado no Gráfico 1.

Gráfico 1. Curva de acumulação de espécies utilizada na determinação da suficiência amostral em levantamento etnobotânico e etnofarmacológico realizado junto à comunidade da área de abrangência da USF Itambezinho (Campo Largo/PR, dez. 2015 a jul. 2016)

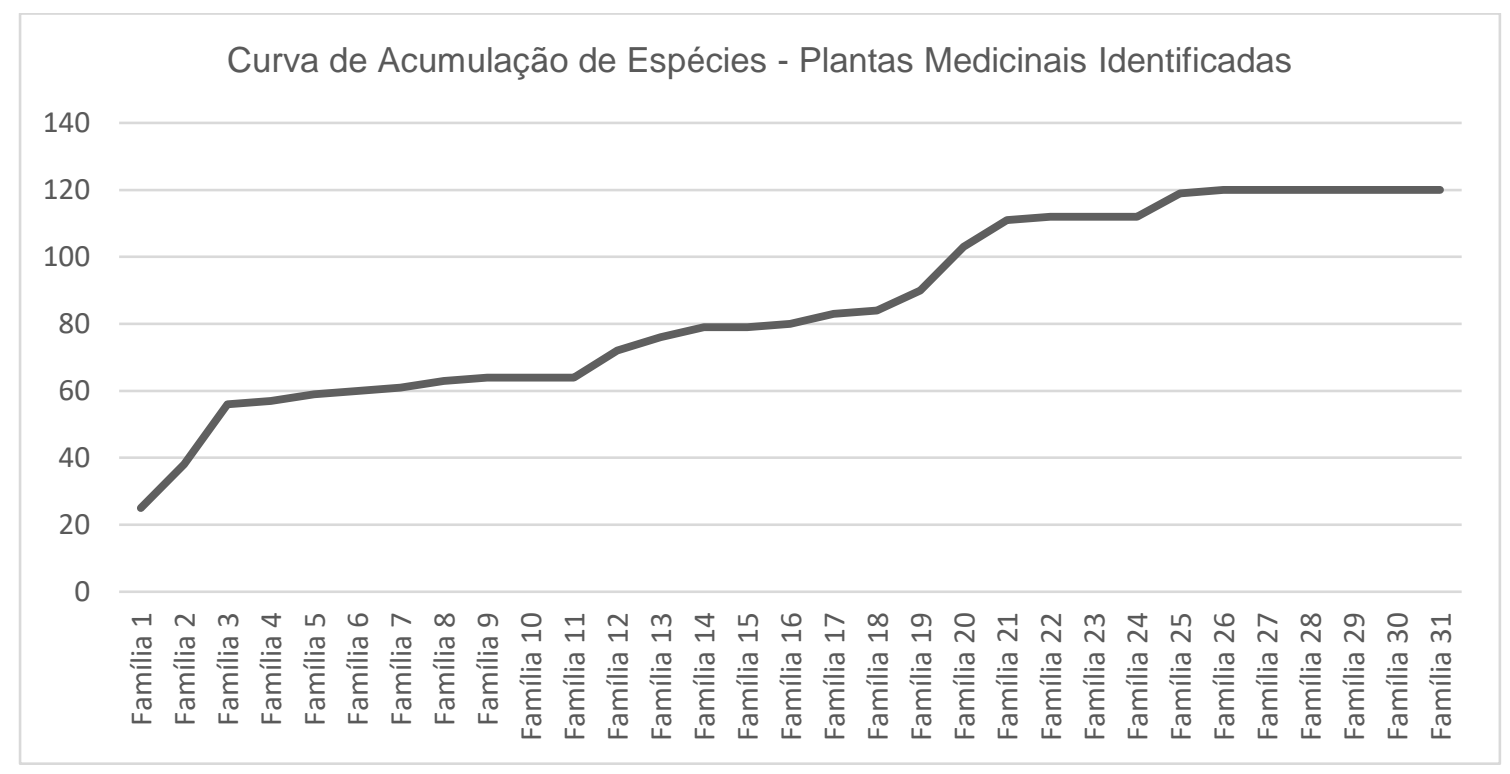

Fonte: Adaptado de GONÇALVES, 2017.

\section{CONCLUSÃO}

Com base nos dados apresentados, demonstramos que a curva de acumulação de espécie constitui um importante método a ser empregado na suficiência amostral em estudos etnobotânicos e etnofarmacológicos. Considerando a rica biodiversidade do Brasil, e o uso de distintos recursos vegetais em diferentes comunidades, a curva apresenta grande relevância para identificação representativa de plantas medicinais nestas comunidades. 


\section{REFERÊNCIAS}

ALBUQUERQUE, U.P.; HANAZAKI, N. As pesquisas etnodirigidas na descoberta de novos fármacos de interesse médico e farmacêutico: fragilidades e pespectivas. Rev. Bras. Farmacogn., 16(Supl.): dez. 2006.

BATISTA, J.L.F.; SCHILLING, A.C. Um Algoritmo Matricial para Construção da "Curva de Acumulação de Espécies". METRVM, n.3, abr. 2006. Disponível em: <http://cmq.esalq.usp.br/wiki/lib/exe/fetch.php?media=publico:metrvm:metrvm-2006n03.pdf>. Acesso em: 23 out. 2017.

BRASIL. Ministério da Saúde. Secretaria de Ciência, Tecnologia e Insumos Estratégicos. Departamento de Assistência Farmacêutica. Política nacional de plantas medicinais e fitoterápicos. Brasília: Ministério da Saúde, 2006a. 60 p. - (Série B. Textos Básicos de Saúde).

BRASIL. Ministério da Saúde. Secretaria de Ciência, Tecnologia e Insumos Estratégicos. Departamento de Assistência Farmacêutica. A fitoterapia no SUS e o Programa de Pesquisa de Plantas medicinais da Central de Medicamentos. Brasília: Ministério da Saúde, 2006b - (Série B. Textos Básicos de Saúde).

BRASIL. Ministério da Saúde. Secretaria de Atenção à Saúde. Departamento de Atenção Básica. Política Nacional de Práticas Integrativas e Complementares no SUS: atitude de ampliação de acesso. 2. ed. Brasília: Ministério da Saúde, 2015; 96 p. il.

DI STASI, L.C. An integrated approach to identification and conservation of medicinal plants in the tropical forest-a Brazilian experience. Plant Genetic Resources, 3(2); 199-205, 2005.

ELISABETSKY, E. Etnofarmacologia. Cienc. Cult., v.55, n.3, São Paulo, July/Sept. 2003.

FIDALGO, O.; BONONI, V.L.R. Técnica de coleta, preservação e herborização de material botânico. (Série Documentos) São Paulo. 62p. 1989.

GIL, A.C. Como elaborar projetos de pesquisa. 4. ed. São Paulo: Atlas, 2002. 
GOMIDE, L.R.; SCOLFORO, J.R.S.; THIERSCH, C.R.; OLIVEIRA, A.D. Uma nova abordagem para definição da suficiência amostral em fragmentos florestais nativos. Cerne, Lavras, v.11, n.4, p.376-388, out./dez. $2005 . \quad$ Disponível em: <http://www.redalyc.org/articulo.oa?id=74411407>. Acesso em: 23 out. 2017.

GOTELLI, N.J.; COLWELL, R.K. Estimating species richness. p.39-54 In: Biological Diversity: Frontiers In Measurement And Assessment. MAGURRAN, A.E.; MCGILL, B.J. (eds.). Oxford University Press, Oxford, 2010. 345 p. Disponível em: <http://www.uvm.edu/ ngotelli/manuscriptpdfs/Chapter\%204.pdf>. Acesso em: 23 out. 2017.

GONÇALVES, R.N. Práticas integrativas na atenção primária à saúde, sob a ótica da fitoterapia. Dissertação (Mestrado). Programa de Pós-Graduação em Saúde Coletiva. Setor de Ciências da Saúde. Universidade Federal do Paraná. Curitiba, 2017. 192 f.

KERSTEN, R.A.; GALVÃO, F. Suficiência amostral em inventários florísticos e fitossociológicos. In: Fitossociologia no Brasil: métodos e estudos de caso. 1 ed., FELFILI-FAGG, J.M.; EISENLOHR, P.V.; MELO, M.M.R.F.; ANDRADE, L.A.; MEIRA NETO, J.A.A. (eds.). Editora da Universidade Federal de Viçosa, 2011, p.156-173. Disponível em: $<$ https://www.researchgate.net/publication/216755255_Suficiencia_amostral_em_inventari os_floristicos_e_fitossociologicos>. Acesso em: 23 out. 2017.

INFOPÉDIA. Dicionário infopédia de Termos Médicos [em linha]. Porto: Porto Editora, 2003-2017. Disponível em: <https://www.infopedia.pt/dicionarios/termos-medicos>. Acesso em: 05 jun. 2017.

LIMA, L.O.; GOMES, E.C. Alimento ou medicamento? Espécies vegetais frente à legislação brasileira. Rev. Bras. PI. Med., Campinas, v.16, n.3, supl. I, p.771-782, 2014.

MACIEL, M.A.M.; PINTO, A.C.; VEIGA JR., V.F.; GRYNBERG, N.F.; ECHEVARRIA, A. Plantas medicinais: a necessidade de estudos multidisciplinares. Quim. Nova, v.25, n.3, p.429-438, 2002.

SCHILLING, A.C.; BATISTA, J.L.F. Curva de acumulação de espécies e suficiência amostral em florestas tropicais. Revista Brasil. Bot., v.31, n.1, p.179-187, jan.-mar. 2008. 
SCHILLING, A.C.; BATISTA, J.L.F.; COUTO, H.Z. Ausência de estabilização da curva de acumulação de espécies em florestas tropicais. Ciência Florestal, Santa Maria, v.22, n.1, p.101-111, jan.-mar., 2012.

SILVA, D.R.O. Estimativa de riqueza de macroinvertebrados bentônicos e a relação da composição de comunidades com componentes de meso-habitat em riachos de cabeceira no cerrado. Dissertação (Mestrado). Universidade Federal de Minas Gerais, Programa de Pós-graduação em Ecologia, Conservação e Manejo de Vida Silvestre, Belo Horizonte, MG, 2012. $65 \mathrm{f}$.

SILVEIRA, L.F.; BEISIEGEL, B.M.; CURCIO, F.F.; VALDUJO, P.H.; DIXO, M.; VERDADE, V.K.; MATTOX, G.M.T.; CUNNINGHAM, P.T.M. Para que servem os inventários de fauna? Estudos Avançados, v.24, n.68, p.173-207, 2010.

WANDSCHEER, A.C.D.; BAUMART, J.S.; MARCHESAN, E.; SANTOS, S.; AVILA, L.A.; SOARES, C.F.; PIRES, M.M. Suficiência amostral para estudos de impacto ambiental sobre a comunidade de macroinvertebrados bentônicos em arrozais irrigados. Ciência Rural, Santa Maria, v.46, n.1, p.26-29, jan, 2016. 